\title{
Connecting neutrino masses and dark matter by high-dimensional lepton number violation operator
}

\author{
Chao-Qiang Geng, ${ }^{a, b, c}$ Da Huang, ${ }^{b}$ Lu-Hsing Tsai ${ }^{b}$ and Qing Wang ${ }^{d, e}$ \\ ${ }^{a}$ Chongqing University of Posts \& Telecommunications, \\ Chongqing, 400065, China \\ ${ }^{b}$ Department of Physics, National Tsing Hua University, \\ Hsinchu, 300 Taiwan \\ ${ }^{c}$ Physics Division, National Center for Theoretical Sciences, \\ Hsinchu, 300 Taiwan \\ ${ }^{d}$ Department of Physics, Tsinghua University, \\ Beijing 100084, China \\ ${ }^{e}$ Collaborative Innovation Center of Quantum Matter, \\ Beijing 100084, China \\ E-mail: geng@phys.nthu.edu.tw, dahuang@phys.nthu.edu.tw, \\ lhtsai@phys.nthu.edu.tw, wangq@mail.tsinghua.edu.cn
}

ABSTRACT: We propose a new model with the Majorana neutrino masses generated at two-loop level, in which the lepton number violation (LNV) processes, such as neutrinoless double beta decays, are mainly induced by the dimension-7 LNV effective operator $\mathcal{O}_{7}=\bar{l}_{R}^{c} \gamma^{\mu} L_{L}\left(D_{\mu} \Phi\right) \Phi \Phi$. Note that it is necessary to impose an $Z_{2}$ symmetry in order that $\mathcal{O}_{7}$ dominates over the conventional dimension-5 Weinberg operator, which naturally results in a stable $Z_{2}$-odd neutral particle to be the cold dark matter candidate. More interestingly, due to the non-trivial dependence of the charged lepton masses, the model predicts the neutrino mass matrix to be in the form of the normal hierarchy. We also focus on a specific parameter region of great phenomenological interests, such as electroweak precision tests, dark matter direct searches along with its relic abundance, and lepton flavor violation processes.

Keywords: Neutrino Physics, Global Symmetries, Effective field theories

ARXIV EPRINT: 1507.03455 


\section{Contents}

1 Introduction 1

2 Generation of neutrino masses and $0 \nu \beta \beta$ decays 2

2.1 The model 2

2.2 Two-loop Majorana neutrino masses 3

$\begin{array}{lll}2.3 & \text { Neutrinoless double beta decay } & 6\end{array}$

3 Phenomenological constraints $\quad 7$

$\begin{array}{lll}3.1 & \text { Electroweak precision tests } & 7\end{array}$

$\begin{array}{llr}3.2 & \text { Dark matter } & 8\end{array}$

3.3 Lepton flavor violation $\quad 9$

$\begin{array}{lll}3.4 & \text { Numerical results } & 10\end{array}$

4 Conclusions 11

\section{Introduction}

The presence of the tiny neutrino masses and mixings between different neutrino flavors have been established by many neutrino oscillation experiments [1-7], while more and more evidences are accumulated for the existence of dark matter (DM) over the last several decades, with the most precise measurement of its relic abundance by PLANCK [8, 9]. Both phenomena cannot be explained within the Standard Model (SM), thus providing us with two windows towards new physics beyond it. An interesting idea is to connect neutrinos and DM in a unified framework as many existing attempts in the literature (see e.g. refs. [10-13]). We would like to push this connection further in the present paper.

In order to understand the mass hierarchy problem in the neutrino sector, there are many models in the literature to naturally generate small Majorana neutrino masses such as the traditional Seesaw [14-26] and radiative mass generation mechanisms [10-13, 27-29]. Most of them can be summarized as a specific realization of the conventional dimension- 5 Weinberg operator. However, the generation of Majorana neutrino masses only requires the lepton number violation (LNV) by two units, and there exist many other equally legitimate LNV effective operators [30-38], which are composed of the SM fields but with higher scaling dimensions. From the effective field theory perspective, it is generically believed that these high-dimensional effective operators are subdominated by the Weinberg operator due to the suppression from the corresponding high powers of the large cutoff. In order for these operators to show up as the leading contributions, one usually needs to impose an additional symmetry on the model to break the usual scaling arguments. Furthermore, if this symmetry is kept unbroken, then the lightest symmetry-protected neutral particle would provide a perfect DM candidate. In this way, the symmetry connects 


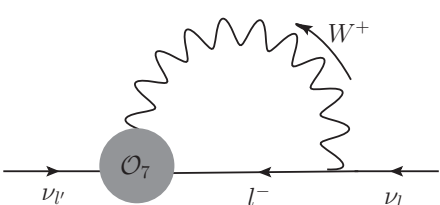

(a)

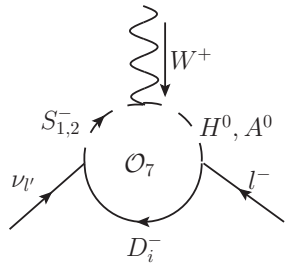

(b)

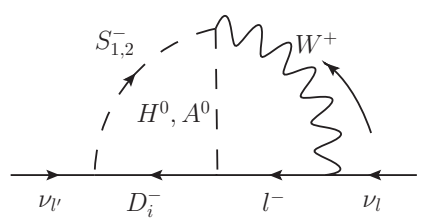

(c)

Figure 1. Typical diagrams for (a) induced neutrino masses by the effective operator $\mathcal{O}_{7}$, (b) the one-loop realization of $\mathcal{O}_{7}$, and (c) the two-loop neutrino mass generation.

Majorana neutrino masses and DM physics by the high-dimensional effective operators. Such a connection has been already exemplified by some recent three-loop neutrino mass models $[13,39-41]$, which realize the dimension-9 effective operator $\mathcal{O}_{9}=\bar{l}^{c}{ }_{R} l_{R}\left[\left(D_{\mu} \Phi\right) \Phi\right]^{2}$ with the DM embedded in the loop.

In this study, we focus on a specific dimension-7 LNV operator $\mathcal{O}_{7}=\bar{l}_{R}^{c} \gamma^{\mu} L_{L}\left(D_{\mu} \Phi\right) \Phi \Phi$ $[32,34,37]$ and construct a UV complete model with an unbroken $Z_{2}$ symmetry to accomplish the above general arguments. In this model, Majorana neutrino masses arise radiatively at two-loop level, and neutrinoless double beta $(0 \nu \beta \beta)$ decays are dominated by a new "long-range" contribution, ${ }^{1}$ as the results of the existence of $\mathcal{O}_{7}$, while DM can also be embedded naturally as the lightest $Z_{2}$-odd neutral state.

This paper is organized as follows. In section 2, we first describe the particle content and write down the relevant part of the Lagrangian for the model. We then calculate the two-loop neutrino masses and new contributions to the $0 \nu \beta \beta$ decay rate in the model, with the emphasis on their relations to the high-dimensional effective operator $\mathcal{O}_{7}$. In section 3 , the constraints on the model are addressed from the electroweak (EW) precision tests, dark matter searches, and lepton flavor violating (LFV) processes. Finally, we give the conclusions in section 4 .

\section{Generation of neutrino masses and $0 \nu \beta \beta$ decays}

\section{$2.1 \quad$ The model}

Figure 1a shows the neutrino mass generation induced by the one-loop diagram with $\mathcal{O}_{7}$. In order to produce $\mathcal{O}_{7}$ at one-loop level, we introduce two scalars: $s:(1,2)$ and $\chi:(2,1 / 2)$, and three vector-like fermions $D_{(L, R) i}:(2,1 / 2)$ with $i=1,2$ and 3 to the SM under $\mathrm{SU}(2)_{L} \times \mathrm{U}(1)_{Y}$. A $Z_{2}$ symmetry is also imposed, in which only the new particles carry odd charges. The relevant new parts of the Lagrangian are given by

$$
\begin{aligned}
-\Delta L=\mu_{s}^{2}\left(s^{*} s\right)+\mu_{\chi}^{2}\left(\chi^{\dagger} \chi\right) & +V_{4}+\left[\xi_{i l} \overline{\left(D_{L i}\right)^{c}}\left(i \sigma_{2}\right) L_{L l} s+\zeta_{i l} \overline{\left(D_{L i}\right)} l_{R l} \chi+M_{D i} \bar{D}_{L i} D_{R i}\right. \\
& \left.+\kappa s^{*} \chi^{\dagger}\left(i \sigma_{2}\right) \Phi+\frac{\lambda_{5}}{2}\left(\chi^{\dagger} \Phi\right)^{2}+\text { H.c. }\right] \\
V_{4}=\lambda_{3}\left(\Phi^{\dagger} \Phi\right)\left(\chi^{\dagger} \chi\right)+ & \lambda_{4}\left(\Phi^{\dagger} \chi\right)\left(\chi^{\dagger} \Phi\right)+\lambda_{\Phi s}\left(\Phi^{\dagger} \Phi\right)\left(s^{*} s\right)+\lambda_{\chi s}\left(\chi^{\dagger} \chi\right)\left(s^{*} s\right) \\
+ & \lambda_{\chi}\left(\chi^{\dagger} \chi\right)^{2}+\lambda_{s}\left(s^{*} s\right)^{2}
\end{aligned}
$$

\footnotetext{
${ }^{1}$ The definitions of "short" and "long" range contributions to $0 \nu \beta \beta$ follow refs. [42, 43].
} 
where $\Phi=\left(\Phi^{+}, \Phi^{0}\right)^{T}$ is the SM Higgs doublet and $\sigma_{2}$ is the Pauli matrix for the $\mathrm{SU}(2)_{L}$ gauge group. After the EW spontaneous symmetry breaking, $\Phi$ acquires a vacuum expectation value $v \equiv \sqrt{2}\left\langle\Phi^{0}\right\rangle \simeq 246 \mathrm{GeV}$, while $\mu_{\chi}^{2}>0$ is necessary for preserving the $Z_{2}$ symmetry. Notice that the lepton number is explicitly broken only when $\kappa, \lambda_{5}$, and at least one of $\xi_{i l} \zeta_{i l^{\prime}}$ are non-zero simultaneously. For convenience, we define $M_{s}^{2}=\mu_{s}^{2}+\frac{1}{2} \lambda_{\Phi s} v^{2}$ and $M_{\chi}^{2}=\mu_{\chi}^{2}+\frac{1}{2} \lambda_{3} v^{2}$. The trilinear coupling constant $\kappa$ in eq. (2.1) makes $s^{ \pm}$mix with $\chi^{ \pm}$, which can be formulated as

$$
\left(\begin{array}{c}
s^{ \pm} \\
\chi^{ \pm}
\end{array}\right)=\left(\begin{array}{cc}
c_{\theta} & -s_{\theta} \\
s_{\theta} & c_{\theta}
\end{array}\right)\left(\begin{array}{c}
S_{1}^{ \pm} \\
S_{2}^{ \pm}
\end{array}\right), t_{2 \theta}=\frac{\sqrt{2} \kappa v}{M_{s}^{2}-M_{\chi}^{2}},
$$

where $s_{x} \equiv \sin x, c_{x} \equiv \cos x, t_{x} \equiv \tan x$, and $S_{1}^{ \pm}$and $S_{2}^{ \pm}$represent the charged mass eigenstates with their masses, given by

$$
\begin{aligned}
& M_{S_{1}}^{2}=\frac{c_{\theta}^{2}}{c_{2 \theta}} M_{s}^{2}-\frac{s_{\theta}^{2}}{c_{2 \theta}} M_{\chi}^{2}, \\
& M_{S_{2}}^{2}=\frac{c_{\theta}^{2}}{c_{2 \theta}} M_{\chi}^{2}-\frac{s_{\theta}^{2}}{c_{2 \theta}} M_{s}^{2},
\end{aligned}
$$

respectively. On the other hand, $\lambda_{5}$ contributes to the mass splitting between $H^{0}$ and $A^{0}$ in $\chi$, shown as

$$
M_{H}^{2}=M_{\chi}^{2}+\frac{1}{2}\left(\lambda_{4}+\lambda_{5}\right) v^{2}, M_{A}^{2}=M_{\chi}^{2}+\frac{1}{2}\left(\lambda_{4}-\lambda_{5}\right) v^{2} .
$$

As for the new fermions, we have the tree-level relation $M_{D_{i}^{ \pm}}=M_{D_{i}^{0}}$ for each $D_{i}$ doublet. The mass splittings between the charge and neutral components of the inert fermion doublets can only be induced by loop corrections with values around a few hundred $\mathrm{MeV}$ [44].

In this paper, we will characterize the model by using the physical quantities:

$$
M_{H}, M_{A}, M_{S_{1}}, M_{S_{2}}, M_{D_{i}}, s_{\theta}, \lambda_{L}, \xi_{i l}, \text { and } \zeta_{i l},
$$

where $\lambda_{L} \equiv \frac{1}{2}\left(\lambda_{3}+\lambda_{4}+\lambda_{5}\right)$, and the other independent coupling constants from quarter terms:

$$
\lambda_{\Phi s}, \lambda_{\chi s}, \lambda_{\chi} \text {, and } \lambda_{s}
$$

which are less relevant in our discussion.

\subsection{Two-loop Majorana neutrino masses}

As seen in figure $1 \mathrm{~b}$, the effective operator $\mathcal{O}_{7}$ can be induced by the one-loop diagram, whereas the Weinberg operator cannot. ${ }^{2}$ Consequently, Majorana neutrino masses appear

${ }^{2}$ There is a similar realization of $\mathcal{O}_{7}$ in ref. [37], in which a triplet replaced the singlet $s$ of our model. A fundamental distinction of their paper from the present one is that $\mathcal{O}_{7}$ does not give the dominant contribution to Majorana neutrino masses in ref. [37]. 
through the two-loop diagram in figure $1 \mathrm{c} .^{3}$ The resulting neutrino mass matrix $M_{\nu}$ defined in the Lagrangian $-\frac{1}{2} \overline{\left(\nu_{L}^{c}\right)_{l}}\left(M_{\nu}\right)_{l l^{\prime}}\left(\nu_{L}\right)_{l^{\prime}}+$ H.c. can be calculated as

$$
\left(M_{\nu}\right)_{l l^{\prime}}=-\frac{1}{\left(16 \pi^{2}\right)^{2}} \frac{G_{F} s_{2 \theta}}{\sqrt{2}}\left(M_{H}^{2}-M_{A}^{2}\right) \sum_{i=1,2,3}\left(m_{l} \zeta_{i l} \xi_{i l^{\prime}}+m_{l^{\prime}} \zeta_{i l^{\prime}} \xi_{i l}\right)\left[I_{i 1}-I_{i 2}\right]
$$

where $m_{l}(l=e, \mu, \tau)$ are charged lepton masses, and $I_{i j}$ are defined by

$$
\begin{aligned}
I_{i j} \equiv \int_{0}^{1} d y_{2} \int_{0}^{1-y_{2}} d y_{1} \int_{0}^{1} d x_{3} \int_{0}^{1-x_{3}} d x_{2} \int_{0}^{1-x_{2}-x_{3}} d x_{1} \\
\quad\left\{\left[\frac{2(1-3 x)}{x(1-x)}+\frac{6 y_{1}(2-x)}{(1-x)^{2}}\right] \log \left(m_{i j}^{2}\right)+\frac{-2 y_{1}(2-x)}{x(1-x)} \frac{M_{W}^{2}}{m_{i j}^{2}}\right\}, \\
m_{i j}^{2} \equiv y_{1}\left[x_{1} M_{H}^{2}+x_{2} M_{A}^{2}+x_{3} M_{W}^{2}\right]+y_{2} x(1-x) M_{S_{j}}^{2} \\
\quad+\left(1-y_{1}-y_{2}\right) x(1-x) M_{D i}^{2}, \\
x=x_{1}+x_{2} .
\end{aligned}
$$

Subsequently, one can diagonalize $M_{\nu}$ by

$$
\operatorname{diag}\left(m_{1}, m_{2}, m_{3}\right)=V^{T} M_{\nu} V
$$

where $m_{1,2,3}$ are three neutrino mass eigenvalues, which can have the normal ordering, $m_{1}<m_{2} \ll m_{3}$, or inverted ordering, $m_{3} \ll m_{1}<m_{2}$, and $V$ is the Pontecorvo-MakiNakagawa-Sakata mixing matrix $[47,48]$. Without loss of generality, $V$ can be written as the standard parametrization by appropriate rephasing in $L_{L}$ 's and $l_{R}$ 's, given by [49]

$$
V=\left(\begin{array}{ccc}
c_{12} c_{13} & s_{12} c_{13} & s_{13} e^{-i \delta} \\
-s_{12} c_{23}-c_{12} s_{23} s_{13} e^{i \delta} & c_{12} c_{23}-s_{12} s_{23} s_{13} e^{i \delta} & s_{23} c_{13} \\
s_{12} s_{23}-c_{12} c_{23} s_{13} e^{i \delta} & -c_{12} s_{23}-s_{12} c_{23} s_{13} e^{i \delta} & c_{23} c_{13}
\end{array}\right)\left(\begin{array}{ccc}
1 & 0 & 0 \\
0 & e^{i \alpha_{21} / 2} & 0 \\
0 & 0 & e^{i \alpha_{31} / 2}
\end{array}\right),
$$

where the mixing angles with $s_{i j} \equiv \sin \theta_{i j}$ and $c_{i j} \equiv \cos \theta_{i j}$ are defined within $[0, \pi / 2]$, and Dirac phase $\delta$ and Majorana phases $\alpha_{21}$ and $\alpha_{31}$ are defined within [0,2 $]$.

From eq. (2.8), we can get two important features for this mass generation mechanism. Firstly, the overall size of $M_{\nu}$ is proportional to the mass difference of the neutral scalars, $M_{H}^{2}-M_{A}^{2}$, and the combined factor of the charged states, $s_{2 \theta}\left(I_{i 1}-I_{i 2}\right)$, in which the former is generated by $\lambda_{5}$ and the latter corresponds to the size of $\kappa$. Turning off one of them will make all neutrinos massless. Secondly, the neutrino masses are positively correlated to the coupling matrix elements $\xi_{i l}$ and $\zeta_{i l}$, as well as the sizes of $m_{l}$. As the existence of the charged lepton mass hierarchy, $m_{e} \ll m_{\mu}<m_{\tau}$, if both matrices of $\left(\xi_{i l}\right)$ and $\left(\zeta_{i l}\right)$ are in uniform textures, the magnitude of $\left(M_{\nu}\right)_{e e}$ should be much smaller than those of other $M_{\nu}$ entries. We make a great advantage of this general expectation by taking the following limit

$$
\left(M_{\nu}\right)_{e e} \simeq 0
$$

\footnotetext{
${ }^{3}$ Similar topology with one $W^{ \pm}$exchange in a two-loop neutrino mass model can also be found in refs. $[45,46]$, in which a different high-dimensional effective operator is realized without DM.
} 
which is shown in refs. $[49,50]$ to rule out the inverted ordering of neutrino mass spectrum at more than $2 \sigma$ confident level. Thus, the normal ordering is predicted for the present model. Note that in the limit of eq. (2.13), ref. [49] even shows that the lightest neutrino mass $m_{1}$ can only be located within the range $0.001 \mathrm{eV} \lesssim m_{1} \lesssim 0.01 \mathrm{eV}$. Moreover, the smallness of $\left(M_{\nu}\right)_{e e}$ is also required by the $0 \nu \beta \beta$ decay, which will be clear in the next subsection.

If we further focus on the CP conserving case, i.e., $\delta, \alpha_{21}, \alpha_{31}=0$ or $\pi$, then $m_{1}$ will be constrained in the two narrow regimes, around 0.002 and $0.007 \mathrm{eV}$ with $\left\{\delta, \alpha_{21}, \alpha_{31}\right\}=$ $\{0, \pi, 0\}$ (Texture A) or $\{\pi, \pi, 0\}$ (Texture B) and $\{0, \pi, \pi\}$ (Texture C) or $\{\pi, \pi, \pi\}$ (Texture D), respectively. Taking the central values of $\theta_{12}, \theta_{23}, \theta_{13}, \Delta m_{21}^{2}$, and $\Delta m_{32}^{2}$ from the global fitting for the neutrino oscillation data [49], the corresponding mass matrices for Textures $\mathrm{A}, \mathrm{B}, \mathrm{C}$, and $\mathrm{D}\left(\mathrm{T}_{\mathrm{A}}, \mathrm{T}_{\mathrm{B}}, \mathrm{T}_{\mathrm{C}}\right.$ and $\left.\mathrm{T}_{\mathrm{D}}\right)$ are given by

$$
\begin{aligned}
& \mathrm{T}_{\mathrm{A}}: M_{\nu}=\left(\begin{array}{ccc}
0 & 0.12 & 0.92 \\
0.12 & 1.9 & 2.7 \\
0.92 & 2.7 & 2.4
\end{array}\right)\left(10^{-2}\right) \mathrm{eV} \\
& \mathrm{T}_{\mathrm{B}}: M_{\nu}=\left(\begin{array}{ccc}
0 & -0.90 & -0.24 \\
-0.90 & 1.7 & 2.7 \\
-0.24 & 2.7 & 2.6
\end{array}\right)\left(10^{-2}\right) \mathrm{eV} \\
& \mathrm{T}_{\mathrm{C}}: M_{\nu}=\left(\begin{array}{ccc}
0 & -1.1 & -0.055 \\
-1.1 & -2.3 & -2.1 \\
-0.055 & -2.1 & -3.1
\end{array}\right)\left(10^{-2}\right) \mathrm{eV} \\
& \mathrm{T}_{\mathrm{D}}: M_{\nu}=\left(\begin{array}{ccc}
0 & -0.086 & 1.1 \\
-0.086 & -2.6 & -2.2 \\
1.1 & -2.2 & -2.9
\end{array}\right)\left(10^{-2}\right) \mathrm{eV}
\end{aligned}
$$

respectively.

We now search for possible coupling matrix forms to realize the above four CP conserving neutrino textures. For simplicity, hereafter we will take $M_{D 1}=M_{D 2}=M_{D 3}=M_{D}$, and also set $\xi$ proportional to the identity matrix with the diagonal matrix element to be $\xi_{d}$. Taking a symmetric form of $\zeta$, the mass matrix element should be proportional to $\xi_{d} \zeta_{l^{\prime} l}\left(m_{l}+m_{l^{\prime}}\right)$. We remark that by an appropriate phase absorption to the fermion fields, one can always have a positive $\xi_{d}$ without loss of generality. Comparing with eqs. (2.14)(2.17), the forms of $\zeta_{l l^{\prime}}$ in the four neutrino matrix textures can be obtained as

$$
\begin{array}{ll}
\mathrm{T}_{\mathrm{A}}: \zeta \propto\left(\begin{array}{ccc}
\times & 0.12 & 0.052 \\
0.12 & 0.89 & 0.14 \\
0.052 & 0.14 & 0.068
\end{array}\right), & \mathrm{T}_{\mathrm{B}}: \zeta \propto\left(\begin{array}{ccc}
\times & -0.84 & -0.013 \\
-0.84 & 0.82 & 0.14 \\
-0.013 & 0.14 & 0.072
\end{array}\right), \\
\mathrm{T}_{\mathrm{C}}: \zeta \propto\left(\begin{array}{ccc}
\times & -1 . & -0.0031 \\
-1 . & -1.1 & -0.11 \\
-0.0031 & -0.11 & -0.088
\end{array}\right), & \mathrm{T}_{\mathrm{D}}: \zeta \propto\left(\begin{array}{ccc}
\times & -0.081 & 0.062 \\
-0.081 & -1.2 & -0.12 \\
0.062 & -0.12 & -0.081
\end{array}\right),
\end{array}
$$

where the cross means that the value of $\zeta_{e e}$ is still arbitrary at this stage, which will be constrained by $0 \nu \beta \beta$ decays. The overall scale of $\zeta_{l l^{\prime}}$ can be determined by eq. (2.8) when 


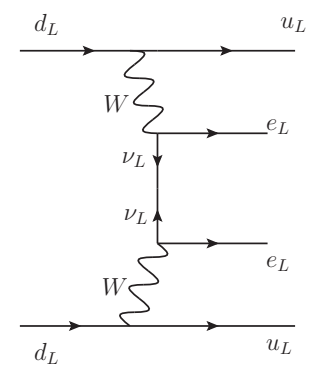

$(a)$

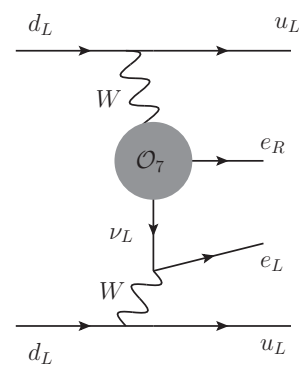

$(b)$

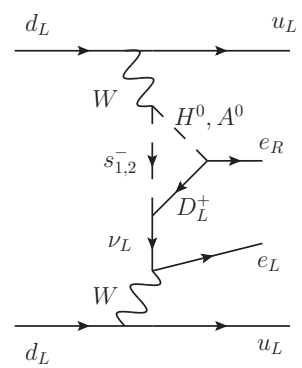

$(c)$

Figure 2. Diagrams for $0 \nu \beta \beta$ decays from (a) the traditional processes with the neutrino mass insertion on the propagator, (b) the contribution involving $\mathcal{O}_{7}$, and (c) the one-loop construction which realizes $\mathcal{O}_{7}$. For (b) and (c), the corresponding upside-down diagrams also need be considered.

all new particle masses, $s_{\theta}$ and $\xi_{d}$ are known. We will also leave the discussion about the correlation between $\xi$ and $\zeta$ from the LFV constraints to section 3 .

\subsection{Neutrinoless double beta decay}

In the previous section, we have built a two-loop neutrino mass model in which the LNV operator $\mathcal{O}_{7}$ provides the leading contribution. The next step is to study the LNV effect in this model induced by this high-dimensional operator. The most sensitive smoking gun for the LNV is the $0 \nu \beta \beta$ decay, which will place the strong constraint on $\xi_{d} \zeta_{e e}$ and $\left(M_{\nu}\right)_{e e}$.

For the Majorana neutrino masses, there always exists the traditional long-range decay process by the exchange of neutrinos with a pair of left-handed electrons emitted as shown in figure 2a. Note that there is a chirality flipping on the internal neutrino propagator, which leads to the proportionality of the amplitude to the neutrino mass matrix element $\left(M_{\nu}\right)_{e e}$, in the sense that the detection for $0 \nu \beta \beta$ processes could help to determine or constrain $\left|\left(M_{\nu}\right)_{e e}\right|$. However, it is known that in some types of neutrino models, figure $2 \mathrm{a}$ does not give the main part of this process, and one should have a prior consideration on the effects of other new diagrams. For example, a class of neutrino models [51-53] that can be characterized by the dimension-9 operator $\mathcal{O}_{9}=\bar{l}_{R}^{c} l_{R}\left[\left(D_{\mu} \Phi\right)^{T}\left(i \sigma_{2}\right) \Phi\right]\left[\left(D^{\mu} \Phi\right)^{T}\left(i \sigma_{2}\right) \Phi\right]$ is well studied in refs. [51, 52, 54-56], and the new contribution is much larger than that from figure $2 \mathrm{a}$ by orders of magnitude of $10^{8}$. For our model or those with $\mathcal{O}_{7}$ as the main LNV source, the $0 \nu \beta \beta$ decays are dominated by the diagram in figure $2 \mathrm{~b}$, which is not suppressed by the nearly-vanishing $\left(M_{\nu}\right)_{e e}$. We can write down a general formula for the half lifetime $T_{1 / 2}^{0 \nu \beta \beta}$ of the $0 \nu \beta \beta$ decay with the contributions from figures $2 \mathrm{a}$ and $2 \mathrm{~b}$, given by [57]

$$
\begin{aligned}
{\left[T_{1 / 2}^{0 \nu \beta \beta}\right]^{-1} } & =C_{m m}\left(\frac{M_{e e}}{m_{e}}\right)^{2}+C_{\eta \eta} \eta^{2}+C_{m \eta}\left(\frac{M_{e e}}{m_{e}}\right) \eta \\
\eta & =-\frac{1}{16 \pi^{2}} \frac{s_{2 \theta} \xi \zeta_{e e}}{4}\left(M_{H}^{2}-M_{A}^{2}\right)\left(I_{1}^{\prime}-I_{2}^{\prime}\right)
\end{aligned}
$$

with

$$
I_{j}^{\prime}=\int_{0}^{1} d x_{3} \int_{0}^{1-x 3} d x_{2} \int_{0}^{1-x_{2}-x_{3}} d x_{1} \frac{1}{x_{1} M_{H}^{2}+x_{2} M_{A}^{2}+x_{3} M_{S_{j}}^{2}+\left(1-x_{1}-x_{2}-x_{3}\right) M_{D}^{2}},
$$




\begin{tabular}{|lcccc|}
\hline & $>T_{\exp }\left(10^{25} \mathrm{yr}\right)$ & $C_{\eta \eta}\left(10^{25} \mathrm{yr}\right)^{-1}$ & $\left|\xi_{d} \zeta_{e e}\right|_{\max }$ & $\left|M_{\nu}\right|_{e e}\left(10^{-2}\right) \mathrm{eV}$ \\
\hline GERDA-1 $\left({ }^{76} \mathrm{Ge}\right)[59]$ & 2.1 & $4.4 \times 10^{-9}$ & $3.6 \times 10^{-4}$ & $<0.017$ \\
KamLAND-Zen $\left({ }^{136} \mathrm{Xe}\right)[60]$ & 1.9 & $8.3 \times 10^{-9}$ & $2.8 \times 10^{-4}$ & $<0.013$ \\
NEMO-3( $\left.{ }^{150} \mathrm{Nd}\right)[62]$ & 0.0018 & $2.9 \times 10^{-7}$ & $1.5 \times 10^{-3}$ & $<0.072$ \\
CUORICINO $\left({ }^{130} \mathrm{Te}\right)[63]$ & 0.3 & $2.3 \times 10^{-8}$ & $4.2 \times 10^{-4}$ & $<0.02$ \\
NEMO-3( $\left.{ }^{82} \mathrm{Se}\right)[64,65]$ & 0.036 & $1.5 \times 10^{-8}$ & $1.5 \times 10^{-3}$ & $<0.07$ \\
NEMO-3( $\left({ }^{100} \mathrm{Mo}\right)[65]$ & 0.11 & $3.5 \times 10^{-8}$ & $5.6 \times 10^{-4}$ & $<0.027$ \\
\hline
\end{tabular}

Table 1. Constraints on $\left|\xi_{d} \zeta_{e e}\right|$ from $0 \nu \beta \beta$ for different nuclei as the targets. The corresponding limitation on $\left|M_{\nu}\right|_{e e}$ are also given.

where $C_{m m}, C_{m \eta}$, and $C_{\eta \eta}$ include the phase space integrations and nuclear matrix elements defined in ref. [57], and $\eta$ is the coupling of the interaction $\left(4 G_{F} / \sqrt{2}\right)\left(\bar{u}_{L} \gamma_{\mu} d_{L}\right)\left(\bar{l}_{R} \gamma^{\mu} \nu_{L}^{c}\right)$, which is originated from the one-loop generation for $\mathcal{O}_{7}$ (in figure $2 \mathrm{c}$ ). By using the numerical results therein and also in ref. [58], we find that the contribution proportional to $C_{\eta \eta}$ is much larger than those to $C_{m m}$ and $C_{m \eta}$.

As given in eq. (2.20) that $\eta$ is proportional to $\xi_{d} \zeta_{e e}$, the upper bound on $\left|\xi_{d} \zeta_{e e}\right|$ can be obtained by comparing with the current experimental sensitivities on $T_{1 / 2}^{0 \nu \beta \beta}$ [59-65] in table 1, where we have used $M_{H}=70 \mathrm{GeV}, M_{A}=95 \mathrm{GeV}, M_{S_{1}}=310 \mathrm{GeV}, M_{S_{2}}=90 \mathrm{GeV}$, $M_{D}=200 \mathrm{GeV}$, and $s_{\theta}=0.1$. The strongest constraint is $\left|\xi_{d} \zeta_{e e}\right|<2.8 \times 10^{-4}$, given by the target nucleus Xe. Finally, the contribution from $\mathcal{O}_{7}$ (proportional to $C_{\eta \eta}$ ) is much larger than that from $\left(M_{\nu}\right)_{e e}$ (proportional to $C_{m m}$ ) by a factor of $\mathcal{O}\left(10^{-4}\right)$, because the latter is greatly suppressed by the factor of $m_{e} / v$. On the other hand, lifting up $\left|\left(M_{\nu}\right)_{e e}\right|$ to the average size of $M_{\nu} \sim 10^{-2} \mathrm{eV}$ will result in the excess of the $0 \nu \beta \beta$ decay rates that conflict with the observations. Table 1 also shows the maximum value of $\left(M_{\nu}\right)_{e e}$ for each nucleus.

Finally, we end this section by mentioning that the role of the $Z_{2}$ symmetry is to make the dimension-7 operator $\mathcal{O}_{7}$ become the dominant contributions to the LNV processes and Majorana neutrino masses. Note that as the quantum numbers of the doublet $(\chi)$ and the singlet $(s)$ scalars are the same as those in the Zee's model [27], the Majorana neutrino masses would be mainly generated by the corresponding one-loop diagrams related to the conventional Weinberg operator if the $Z_{2}$ symmetry is absent. However, with the $Z_{2}$ symmetry, $\mathcal{O}_{7}$ is singled out at 1-loop level, while other LNV effective operators, especially the dimension-5 Weinberg operator, are much suppressed since they would be only induced by higher-loop diagrams. In this way, the $Z_{2}$ symmetry breaks the conventional effective operator ordering based on the scaling dimensions. Other LNV effects, like $0 \nu \beta \beta$ decays, would also change the leading modes accordingly.

\section{Phenomenological constraints}

\subsection{Electroweak precision tests}

As discussed previously, in order to have the two-loop neutrino masses in our model, the non-zero coupling constants $\lambda_{5}$ and $\kappa$ are both required. The former splits the masses between $H^{0}$ and $A^{0}$, and the latter mixes the charged states $\chi^{ \pm}$and $s^{ \pm}$which carry different 


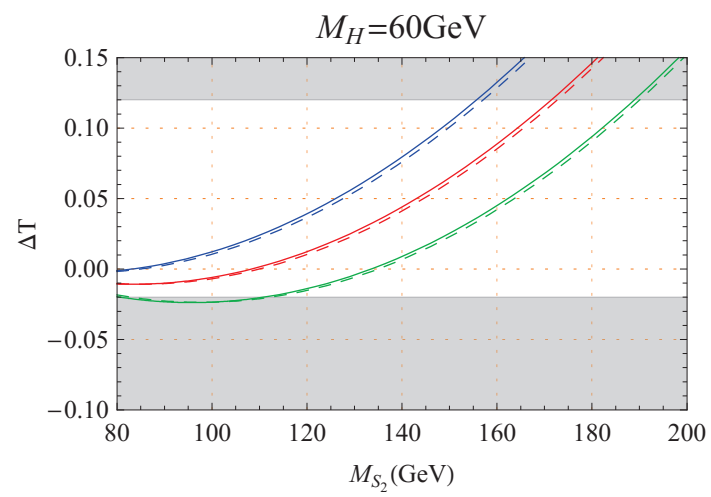

(a)

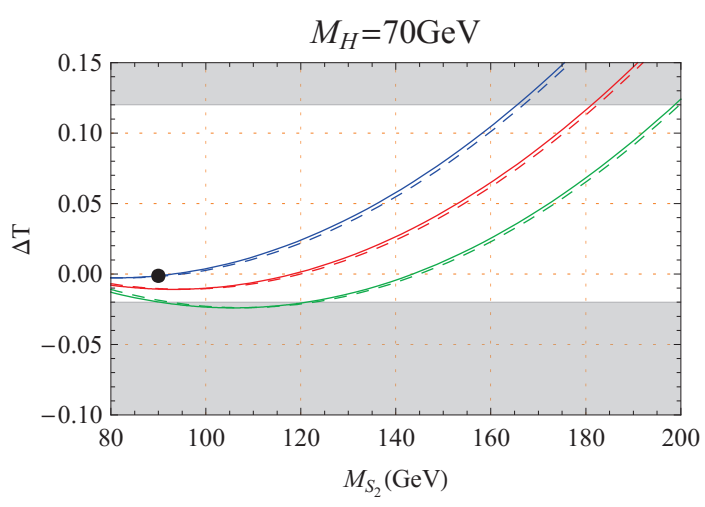

(b)

Figure 3. Plots of $\Delta T$ versus $M_{S_{2}}$ with (a) $M_{H}=60 \mathrm{GeV}$ and (b) $M_{H}=70 \mathrm{GeV}$, where blue, red, and green colors represent $M_{A}-M_{H}=25,50$, and $75 \mathrm{GeV}$, respectively, and solid (dashed) curve corresponds to $s_{\theta}=0.1(0)$, while the black dot is the benchmark point discussed in section 3.D.

EW gauge quantum numbers. Both effects could change the values of the EW oblique $S$ and $T$ parameters. In particular, the $T$ parameter should yield a stronger constraint on this model. The deviation of $T$ from the SM is given by [13]

$\Delta T=\frac{1}{4 \pi s_{W}^{2} M_{W}^{2}}\left[\frac{s_{\theta}^{2}}{4}\left(F_{S_{1}^{ \pm}, H^{0}}+F_{S_{1}^{ \pm}, A^{0}}\right)+\frac{c_{\theta}^{2}}{4}\left(F_{S_{2}^{ \pm}, H^{0}}+F_{S_{2}^{ \pm}, A^{0}}\right)-\frac{1}{2} c_{\theta}^{2} s_{\theta}^{2} F_{S_{1}^{ \pm}, S_{2}^{ \pm}}-\frac{1}{4} F_{H^{0}, A^{0}}\right]$,

with the function $F$ defined by

$$
F_{x, y}=\frac{M_{x}^{2}+M_{y}^{2}}{2}-\frac{M_{x}^{2} M_{y}^{2}}{M_{x}^{2}-M_{y}^{2}} \log \left(\frac{M_{x}^{2}}{M_{y}^{2}}\right) .
$$

The value of $F_{x, y}$ becomes zero when $M_{x} \rightarrow M_{y}$, and it increases with the mass splitting among the new scalars. Note that $\Delta T$ has little to do with $D_{i}$ since there is neither mixing between $D_{i}$ and the SM leptons nor tree-level mass splitting among $D_{i}$, while the deviation for the $S$ parameter can also be ignored [66]. The formulae of eq. (3.1) is a general result for the models with the mixings between the inert doublet and singlet scalars. The global fitting results constrain $\Delta T$ at $1.5-1.7 \sigma$ deviation by $-0.02<\Delta T<0.12$ [49]. We show $\Delta T$ as a function of $M_{S_{2}}$ in figure 3 , where we have used $M_{S_{1}}=310 \mathrm{GeV}$ and $s_{\theta}=0.1(0)$ along with (a) $M_{H}=60$ and (b) $70 \mathrm{GeV}$. It is obvious that the numerical result of our model with $s_{\theta}=0.1$ is approximately equal to a pure inert doublet model with $s_{\theta}=0$. In general, $\Delta T$ goes up with increasing $M_{S_{2}}$, and for a large value of $M_{A}-M_{H}$, the constraint on $M_{S_{2}}$ becomes stronger. The figure also shows that $M_{A}-M_{H}$ is limited to be less than $75 \mathrm{GeV}$ for $M_{H}=60$ to $70 \mathrm{GeV}$ and $90 \lesssim M_{S_{2}} \lesssim 110 \mathrm{GeV}$. Finally, it should be noted that $M_{S_{2}}$ can not be too small, since there exists a lower bound on $M_{S_{2}}$ located within 70 to $90 \mathrm{GeV}$ [67] from the LEP experiments.

\subsection{Dark matter}

In this model, the lightest of the extra neutral particles: $H^{0}, A^{0}$, and $D_{1,2,3}^{0}$ could be a DM candidate, whose stability is guaranteed by the imposed $Z_{2}$ symmetry. In the 
following, we will focus on the case that DM is constituted solely by $H^{0}$ with a small charged scalar mixing $s_{\theta}$, in which our DM would be very similar to that in the wellstudied inert doublet model [68-70]. Furthermore, we concentrate on the low DM mass region with $50 \mathrm{GeV} \leqslant M_{H} \leqslant 80 \mathrm{GeV}[69,70]$, in which a large $H^{0}-A^{0}$ mass splitting can be allowed for the generation of the right two-loop neutrino masses. In addition, the mass of $S_{2}^{ \pm}$should be higher than $90 \mathrm{GeV}$ in order to escape the LEP bounds [67], so that the co-annihilation channels, such as $H^{0}-A^{0}$ and $H^{0}-S_{2}^{ \pm}$, would be strongly suppressed and thus ignored.

We use the package micrOMEGAs [71] to accurately calculate the relic abundance $\Omega_{H}$ in the above parameter space, including all possible annihilations and co-annihilations. When $M_{H}$ approaches the half of the SM Higgs mass $M_{h} / 2 \simeq 62.5 \mathrm{GeV}[72,73]$, the Higgs resonance in the $s$-channel would become prominent, which is characterized by the coupling $\lambda_{L}$ controlling the trilinear vertex $\left(\lambda_{L} v\right) h H^{0} H^{0}$. However, in other regions, the DM annihilation cross section is dominated by the $W W^{(*)}$ mode. Therefore, the correct DM relic abundance is achieved mainly by the balance of the $W W^{(*)}$ and Higgs resonance channels. Figure 4 shows the relevant parameter space in the $M_{H^{-}}\left|\lambda_{L}\right|$ plane, which can give the observed DM abundance $0.112 \lesssim \Omega_{H} h^{2} \lesssim 0.128$ at $3 \sigma$ level [8, 9, 49]. Note that when DM is heavier than $73 \mathrm{GeV}$, the $W W^{(*)}$ channel would give a too large annihilation cross section to accommodate the DM relic abundance [69, 70], which is omitted in figure 4.

The DM $H^{0}$ in this low mass region could be constrained by the DM direct detection experiments. Since we need a relatively large Higgs-mediation annihilation channel to generate DM relic abundance, the Higgs exchange channel can also give rise to sizeable spin-independent signals, with the corresponding DM-nucleon cross section as follows [69]:

$$
\sigma_{H^{0} N}=\frac{m_{r}^{2}}{4 \pi}\left(\frac{\lambda_{L}}{M_{H} M_{h}^{2}}\right)^{2} f^{2} m_{N}^{2}
$$

Currently, the most stringent bound on the spin-independent DM-nucleon cross section is provided by the LUX experiment [74], with the minimum cross section of $7.6 \times 10^{-46} \mathrm{~cm}^{2}$ for a DM mass of $33 \mathrm{GeV}$. It is shown in figure 4 that the LUX experiment has already probed some parameter space required by the DM relic abundance. Especially, the low DM mass region with $M_{H} \leqslant 52 \mathrm{GeV}$ is actually ruled out, as indicated by the shaded area in the plot. However, most parameter spaces are still allowed by LUX.

\subsection{Lepton flavor violation}

The current experimental constraints on LFV processes, such as the radiative decays $l \rightarrow$ $l^{\prime} \gamma[75,76], \mu-e$ conversions [77-80], and three-lepton decays $l \rightarrow l_{1} l_{2} \bar{l}_{3}[81,82]$, are all dominated by one-loop diagrams with $Z_{2}$ odd particles inside. We take $\mu \rightarrow e \gamma$ as an illustration because the current experimental upper bound $\operatorname{Br}\left(\mu^{+} \rightarrow e^{+} \gamma\right)<5.7 \times 10^{-13}$ [75] usually constrains a model in the most stringent way. In our model, we have

$$
\begin{aligned}
\operatorname{Br}\left(\mu^{\mp} \rightarrow e^{\mp} \gamma\right) & =\frac{\Gamma\left(\mu^{\mp} \rightarrow e^{\mp} \gamma\right)}{\Gamma\left(\mu^{\mp} \rightarrow e^{\mp} \nu \bar{\nu}\right)} \\
& =\frac{3 \alpha_{e}}{64 \pi G_{F}^{2}}\left|\sum_{l} \zeta_{l \mu} \zeta_{l e}\right|^{2}\left(s_{\theta}^{2} K_{S_{1}}+c_{\theta}^{2} K_{S_{2}}+\frac{1}{2} K_{H}^{\prime}+\frac{1}{2} K_{A}^{\prime}\right)^{2},
\end{aligned}
$$




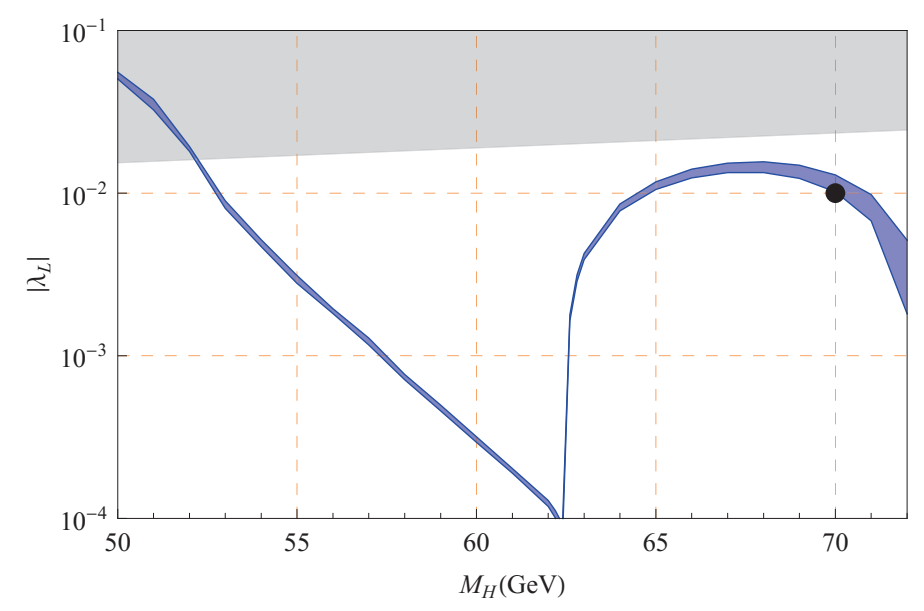

Figure 4. The parameter space for the correction DM relic abundance in the $M_{H}-\left|\lambda_{L}\right|$ plane, with the blue band representing the region within $3 \sigma$ deviation of the cold dark matter relic abundance. The gray shaded area is excluded by the LUX experiment, and the black dot represents the benchmark point. This plot is calculated with $M_{A}=95 \mathrm{GeV}$ and $M_{S_{2}}=90 \mathrm{GeV}$.

where the loop integrals $K_{x}$ and $K_{x}^{\prime}$ are defined as

$$
K_{x}=\frac{2 z^{3}+3 z^{2}-6 z+1-6 z^{2} \log z}{6(1-z)^{4} M_{x}^{2}}, \quad K_{x}^{\prime}=-\frac{z^{3}-6 z^{2}+3 z+2+6 z \log z}{6(1-z)^{4} M_{x}^{2}}
$$

with $z=M_{D}^{2} / M_{x}^{2}$. As is expected, the decay branching ratio is proportional to the coupling constant combination $\left|\sum_{l} \zeta_{l \mu} \zeta_{l e}\right|^{2}$. On the other hand, when the values of $\xi_{d} \zeta_{l l^{\prime}}$ are fixed by the neutrino masses, the only degree of freedom left is the size of $\xi_{d}$. When $\xi_{d}$ is large, $\zeta_{l l^{\prime}}$ should be suppressed, along with all the relevant LFV processes. This feature can be displayed in figure 5 , where $\zeta_{l l^{\prime}}$ are expressed in the forms given in eq. (2.18), with the unknown $\zeta_{e e}$ satisfying the relation $\left|\xi_{d} \zeta_{e e}\right| \lesssim 10^{-4}$, as well as $s_{\theta}=0.1, M_{H}=70, M_{A}=95$, $M_{S_{1}}=310, M_{S_{2}}=90$ and 120 and $M_{D}=200 \mathrm{GeV}$. We find that the texture $T_{C(D)}$ yields the most stringent (weakest) constraint on $\xi_{d}$, such that $\xi_{d} \gtrsim 0.01(0.002)$ is required for $M_{S_{2}}=90 \mathrm{GeV}$. From another angle, if we set $\xi_{d}=0.005$ with $T_{A}$, we can predict that $\operatorname{Br}(\mu \rightarrow e \gamma)=10^{-13}\left(3 \times 10^{-15}\right)$ for $M_{S_{2}}=90(120) \mathrm{GeV}$, which might be measured by the next-generation experiments in the future.

\subsection{Numerical results}

Based on the above constraints from the LFV processes, EW precision tests, direct searches of DM with the required relic abundance, we find a benchmark point from the allowed parameter space, given by:

$$
\begin{aligned}
& M_{H}=70 \mathrm{GeV}, \quad M_{A}=95 \mathrm{GeV}, \quad M_{S_{1}}=310 \mathrm{GeV}, \quad M_{S_{2}}=90 \mathrm{GeV}, \\
& M_{D_{1}}=M_{D_{2}}=M_{D_{3}}=M_{D}=200 \mathrm{GeV}, \quad s_{\theta}=0.1, \quad \lambda_{L}=0.01,
\end{aligned}
$$




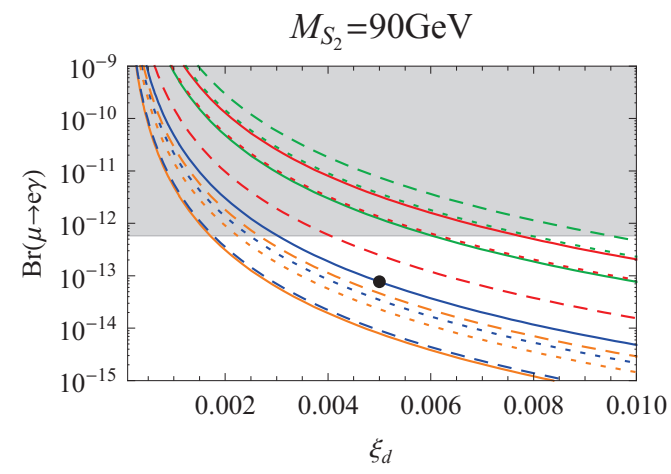

(a)

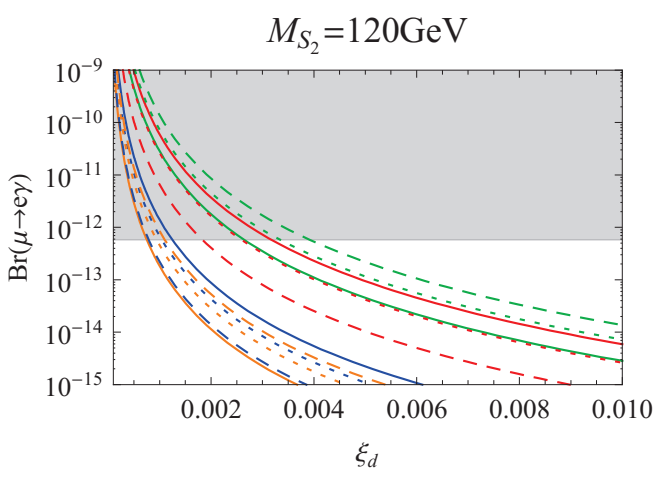

(b)

Figure 5. $\operatorname{Br}(\mu \rightarrow e \gamma)$ versus $\xi$, with (a) $M_{S_{2}}=90 \mathrm{GeV}$ and (b) $M_{S_{2}}=120 \mathrm{GeV}$, where the blue, red, green, and yellow curves correspond to the neutrino textures $T_{A}, T_{B}, T_{C}$, and $T_{D}$, respectively, while the black dot is the benchmark point.

and

$$
\xi=\left(\begin{array}{ccc}
0.005 & 0 . & 0 . \\
0 . & 0.005 & 0 . \\
0 . & 0 . & 0.005
\end{array}\right), \quad \zeta=\left(\begin{array}{ccc}
0.02 & 0.005 & 0.0022 \\
0.005 & 0.038 & 0.0061 \\
0.0022 & 0.0061 & 0.0029
\end{array}\right)
$$

It is clear that the matrix $\zeta$ corresponds to the neutrino texture $T_{A}$. We also plot this benchmark point by a black dot in figures $5 \mathrm{a}, 3 \mathrm{~b}$, and 4 , where the experimental results from LFV processes, oblique parameters, and DM searches are well satisfied, respectively. Finally, from the benchmark point, one can obtain $\lambda_{3}=0.155, \lambda_{4}=-0.067, \lambda_{5}=-0.068$, $\kappa=50.3 \mathrm{GeV}, \mu_{\chi}=65.5 \mathrm{GeV}$, and $M_{S}=309 \mathrm{GeV}$, which are all small enough to ensure the self-consistence of the perturbation theory.

\section{Conclusions}

We have tried to make the connection between neutrino physics and dark matter searches. In particular, we have emphasized that every effective operator, which violates the lepton number by two units, can give an equally good mechanism to generate Majorana neutrino masses. The problem lies in the fact that the new high-dimensional operators might be buried by the overwhelming effects from the conventional Weinberg operator which possess the smallest scaling dimension. One way to break this effective field theory ordering is to impose some symmetry which would protect the lightest neutral symmetry-protected states to be the DM particle.

We have explicitly realized this connection by constructing a UV complete model with the $Z_{2}$ symmetry to generate the dimension-7 operator $\mathcal{O}_{7}=\bar{l}_{R}^{c} \gamma^{\mu} L_{L}\left(D_{\mu} \Phi\right) \Phi \Phi$. We have shown that the Majorana neutrino mass matrix structure and the leading $0 \nu \beta \beta$ decay contribution are closely related to $\mathcal{O}_{7}$. Especially, the neutrino mass matrix is predicted to be of the normal ordering due to the hierarchy in the charged lepton masses, and the $0 \nu \beta \beta$ decay rate can be large enough to be tested in the next-generation experiments. If 
we impose an additional CP symmetry in the lepton sector, we can even determine the form of the neutrino mass matrix completely. We have also focused on a specific parameter region with a small mixing between charged scalars, and considered the constraints from the electroweak precision tests, dark matter searches, and LFV processes.

\section{Acknowledgments}

This work was supported by National Center for Theoretical Sciences, National Science Council (Grant No. NSC-101-2112-M-007-006-MY3) and National Tsing Hua University (Grant No. 104N2724E1), the National Science Foundation of China (NSFC Grant No. 11475092), the Tsinghua University Initiative Scientific Research Program (Grant No. 20121088494).

Open Access. This article is distributed under the terms of the Creative Commons Attribution License (CC-BY 4.0), which permits any use, distribution and reproduction in any medium, provided the original author(s) and source are credited.

\section{References}

[1] GALLEX collaboration, P. Anselmann et al., Implications of the GALLEX determination of the solar neutrino flux., Phys. Lett. B 285 (1992) 390 [INSPIRE].

[2] Super-Kamiokande collaboration, Y. Fukuda et al., Evidence for oscillation of atmospheric neutrinos, Phys. Rev. Lett. 81 (1998) 1562 [hep-ex/9807003] [INSPIRE].

[3] SNO collaboration, Q.R. Ahmad et al., Direct evidence for neutrino flavor transformation from neutral current interactions in the Sudbury Neutrino Observatory,

Phys. Rev. Lett. 89 (2002) 011301 [nucl-ex/0204008] [INSPIRE].

[4] SNO collaboration, Q.R. Ahmad et al., Measurement of day and night neutrino energy spectra at SNO and constraints on neutrino mixing parameters, Phys. Rev. Lett. 89 (2002) 011302 [nucl-ex/0204009] [INSPIRE].

[5] K2K collaboration, M.H. Ahn et al., Measurement of neutrino oscillation by the K2K experiment, Phys. Rev. D 74 (2006) 072003 [hep-ex/0606032] [INSPIRE].

[6] T2K collaboration, K. Abe et al., Indication of electron neutrino appearance from an accelerator-produced off-axis muon neutrino beam, Phys. Rev. Lett. 107 (2011) 041801 [arXiv: 1106.2822] [INSPIRE].

[7] DaYA BAY collaboration, F.P. An et al., Observation of electron-antineutrino disappearance at Daya Bay, Phys. Rev. Lett. 108 (2012) 171803 [arXiv:1203.1669] [INSPIRE].

[8] Planck collaboration, P.A.R. Ade et al., Planck 2013 results. XVI. Cosmological parameters, Astron. Astrophys. 571 (2014) A16 [arXiv: 1303.5076] [INSPIRE].

[9] Planck collaboration, R. Adam et al., Planck 2015 results. I. Overview of products and scientific results, arXiv: 1502.01582 [INSPIRE].

[10] L.M. Krauss, S. Nasri and M. Trodden, A Model for neutrino masses and dark matter, Phys. Rev. D 67 (2003) 085002 [hep-ph/0210389] [INSPIRE].

[11] E. Ma, Verifiable radiative seesaw mechanism of neutrino mass and dark matter, Phys. Rev. D 73 (2006) 077301 [hep-ph/0601225] [INSPIRE]. 
[12] M. Aoki, S. Kanemura and O. Seto, Neutrino mass, dark matter and baryon asymmetry via TeV-scale physics without fine-tuning, Phys. Rev. Lett. 102 (2009) 051805 [arXiv:0807.0361] [INSPIRE].

[13] M. Gustafsson, J.M. No and M.A. Rivera, Predictive model for radiatively induced neutrino masses and mixings with dark matter, Phys. Rev. Lett. 110 (2013) 211802 [arXiv: 1212.4806] [INSPIRE].

[14] P. Minkowski, $\mu \rightarrow$ er at a rate of one out of $10^{9}$ muon decays?, Phys. Lett. B 67 (1977) 421 [INSPIRE].

[15] T. Yanagida, Horizontal symmetry and masses of neutrinos, in the proceedings of the Workshop on unified theory and baryon number in the universe, O. Sawada and A. Sugamoto eds., KEK, Tsukuba, Japan (1979).

[16] M. Gell-Mann, P. Ramond, R. Slansky, Complex spinors and unified theories, in Supergravity, D.Z. Freedman and P.van Nieuwenhuizen eds., North Holland, Amsterdam, The Netherlands (1979).

[17] S.L. Glashow, The future of elementary particle physics, in Quarks and leptons, Cargèse lectures, M. Lévy et al. eds., Plenum Press, New York, U.S.A. (1980).

[18] R.N. Mohapatra and G. Senjanović, Neutrino mass and spontaneous parity violation, Phys. Rev. Lett. 44 (1980) 912 [InSPIRE].

[19] M. Magg and C. Wetterich, Neutrino mass problem and gauge hierarchy, Phys. Lett. B 94 (1980) 61 [INSPIRE].

[20] J. Schechter and J.W.F. Valle, Neutrino masses in $\mathrm{SU}(2) \times \mathrm{U}(1)$ theories, Phys. Rev. D 22 (1980) 2227 [inSPIRE].

[21] T.P. Cheng and L.-F. Li, Neutrino masses, mixings and oscillations in $\mathrm{SU}(2) \times \mathrm{U}(1)$ models of electroweak interactions, Phys. Rev. D 22 (1980) 2860 [INSPIRE].

[22] G.B. Gelmini and M. Roncadelli, Left-handed neutrino mass scale and spontaneously broken lepton number, Phys. Lett. B 99 (1981) 411 [INSPIRE].

[23] G. Lazarides, Q. Shafi and C. Wetterich, Proton lifetime and fermion masses in an $\mathrm{SO}(10)$ model, Nucl. Phys. B 181 (1981) 287 [INSPIRE].

[24] R.N. Mohapatra and G. Senjanović, Neutrino masses and mixings in gauge models with spontaneous parity violation, Phys. Rev. D 23 (1981) 165 [InSPIRE].

[25] J. Schechter and J.W.F. Valle, Neutrino decay and spontaneous violation of lepton number, Phys. Rev. D 25 (1982) 774 [INSPIRE].

[26] R. Foot, H. Lew, X.G. He and G.C. Joshi, Seesaw neutrino masses induced by a triplet of leptons, Z. Phys. C 44 (1989) 441 [inSPIRE].

[27] A. Zee, A theory of lepton number violation, neutrino Majorana mass and oscillation, Phys. Lett. B 93 (1980) 389 [Erratum ibid. B 95 (1980) 461] [INSPIRE].

[28] A. Zee, Quantum numbers of Majorana neutrino masses, Nucl. Phys. B 264 (1986) 99 [INSPIRE].

[29] K.S. Babu, Model of 'calculable' majorana neutrino masses, Phys. Lett. B 203 (1988) 132 [INSPIRE].

[30] K.S. Babu and C.N. Leung, Classification of effective neutrino mass operators, Nucl. Phys. B 619 (2001) 667 [hep-ph/0106054] [INSPIRE].

[31] A. de Gouvêa and J. Jenkins, A survey of lepton number violation via effective operators, Phys. Rev. D 77 (2008) 013008 [arXiv:0708.1344] [INSPIRE]. 
[32] F. del Aguila, A. Aparici, S. Bhattacharya, A. Santamaria and J. Wudka, Effective lagrangian approach to neutrinoless double beta decay and neutrino masses, JHEP 06 (2012) 146 [arXiv:1204.5986] [INSPIRE].

[33] P.W. Angel, N.L. Rodd and R.R. Volkas, Origin of neutrino masses at the LHC: $\Delta L=2$ effective operators and their ultraviolet completions, Phys. Rev. D 87 (2013) 073007 [arXiv: 1212.6111] [INSPIRE].

[34] F. del Águila, A. Aparici, S. Bhattacharya, A. Santamaria and J. Wudka, Neutrinoless double $\beta$ decay with small neutrino masses, PoS(Corfu2012) 028 [arXiv: 1305.4900] [INSPIRE].

[35] P.W. Angel, Y. Cai, N.L. Rodd, M.A. Schmidt and R.R. Volkas, Testable two-loop radiative neutrino mass model based on an LLQd $Q d^{c}$ effective operator, JHEP 10 (2013) 118 [Erratum ibid. 1411 (2014) 092] [arXiv: 1308.0463] [INSPIRE].

[36] Y. Cai, J.D. Clarke, M.A. Schmidt and R.R. Volkas, Testing radiative neutrino mass models at the LHC, JHEP 02 (2015) 161 [arXiv: 1410.0689] [INSPIRE].

[37] A. Aparici, Exotic properties of neutrinos using effective Lagrangians and specific models, arXiv: 1312.0554.

[38] J.C. Helo, M. Hirsch, T. Ota and F.A. P.d. Santos, Double beta decay and neutrino mass models, JHEP 05 (2015) 092 [arXiv: 1502.05188] [INSPIRE].

[39] C.-Q. Geng, D. Huang and L.-H. Tsai, Loop-induced neutrino masses: a case study, Phys. Rev. D 90 (2014) 113005 [arXiv:1410.7606] [inSPIRE].

[40] L.-G. Jin, R. Tang and F. Zhang, A three-loop radiative neutrino mass model with dark matter, Phys. Lett. B 741 (2015) 163 [arXiv:1501.02020] [INSPIRE].

[41] C.-Q. Geng, D. Huang and L.-H. Tsai, Comment on "A three-loop radiative neutrino mass model with dark matter" [Phys. Lett. B 741 (2015) 163], Phys. Lett. B 745 (2015) 56 [arXiv: 1504.05468] [INSPIRE].

[42] F.F. Deppisch, M. Hirsch and H. Pas, Neutrinoless double beta decay and physics beyond the standard model, J. Phys. G 39 (2012) 124007 [arXiv:1208.0727] [InSPIRE].

[43] F. Bonnet, M. Hirsch, T. Ota and W. Winter, Systematic decomposition of the neutrinoless double beta decay operator, JHEP 03 (2013) 055 [Erratum ibid. 1404 (2014) 090] [arXiv: 1212.3045] [INSPIRE].

[44] M. Cirelli and A. Strumia, Minimal dark matter: model and results, New J. Phys. 11 (2009) 105005 [arXiv:0903.3381] [InSPIRE].

[45] K.S. Babu and J. Julio, Two-loop neutrino mass generation through leptoquarks, Nucl. Phys. B 841 (2010) 130 [arXiv:1006.1092] [INSPIRE].

[46] K.S. Babu and J. Julio, Radiative neutrino mass generation through vector-like quarks, Phys. Rev. D 85 (2012) 073005 [arXiv:1112.5452] [INSPIRE].

[47] B. Pontecorvo, Mesonium and anti-mesonium, Sov. Phys. JETP 6 (1957) 429 [Zh. Eksp. Teor. Fiz. 33 (1957) 549] [INSPIRE].

[48] Z. Maki, M. Nakagawa and S. Sakata, Remarks on the unified model of elementary particles, Prog. Theor. Phys. 28 (1962) 870 [InSPIRE].

[49] Particle Data Group collaboration, K. A. Olive et al., Review of particle physics, Chin. Phys. C 38 (2014) 090001 [inSPIRE].

[50] S. Pascoli, S.T. Petcov and L. Wolfenstein, Searching for the CP-violation associated with Majorana neutrinos, Phys. Lett. B 524 (2002) 319 [hep-ph/0110287] [INSPIRE]. 
[51] C.-S. Chen, C.Q. Geng and J.N. Ng, Unconventional neutrino mass generation, neutrinoless double beta decays and collider phenomenology, Phys. Rev. D 75 (2007) 053004 [hep-ph/0610118] [INSPIRE].

[52] C.-S. Chen, C.-Q. Geng, J.N. Ng and J.M.S. Wu, Testing radiative neutrino mass generation at the LHC, JHEP 08 (2007) 022 [arXiv:0706.1964] [INSPIRE].

[53] C.-Q. Geng and L.-H. Tsai, Study of two-loop neutrino mass generation models, arXiv: 1503.06987 [INSPIRE].

[54] F. del Aguila, A. Aparici, S. Bhattacharya, A. Santamaria and J. Wudka, A realistic model of neutrino masses with a large neutrinoless double beta decay rate, JHEP 05 (2012) 133 [arXiv:1111.6960] [INSPIRE].

[55] M. Gustafsson, J.M. No and M.A. Rivera, Radiative neutrino mass generation linked to neutrino mixing and $0 \nu \beta \beta$-decay predictions, Phys. Rev. D 90 (2014) 013012 [arXiv: 1402.0515] [INSPIRE].

[56] S.F. King, A. Merle and L. Panizzi, Effective theory of a doubly charged singlet scalar: complementarity of neutrino physics and the LHC, JHEP 11 (2014) 124 [arXiv:1406.4137] [INSPIRE].

[57] K. Muto, E. Bender and H.V. Klapdor, Nuclear structure effects on the neutrinoless double beta decay, Z. Phys. A 334 (1989) 187 [inSPIRE].

[58] M. Doi, T. Kotani and E. Takasugi, Double beta decay and Majorana neutrino, Prog. Theor. Phys. Suppl. 83 (1985) 1 [INSPIRE].

[59] GERDA collaboration, M. Agostini et al., Results on neutrinoless double- $\beta$ decay of ${ }^{76} G e$ from Phase I of the GERDA experiment, Phys. Rev. Lett. 111 (2013) 122503 [arXiv: 1307.4720] [INSPIRE].

[60] KamLAND-ZEn collaboration, A. Gando et al., Measurement of the double- $\beta$ decay half-life of ${ }^{136}$ Xe with the KamLAND-Zen experiment, Phys. Rev. C 85 (2012) 045504 [arXiv: 1201.4664] [INSPIRE].

[61] KamLAND-Zen collaboration, A. Gando et al., Limit on neutrinoless $\beta \beta$ decay of ${ }^{136} X e$ from the first phase of KamLAND-Zen and comparison with the positive claim in ${ }^{76} \mathrm{Ge}$, Phys. Rev. Lett. 110 (2013) 062502 [arXiv:1211.3863] [INSPIRE].

[62] NEMO collaboration, J. Argyriades et al., Measurement of the double beta decay half-life of Nd-150 and search for neutrinoless decay modes with the NEMO-3 detector, Phys. Rev. C 80 (2009) 032501 [arXiv:0810.0248] [INSPIRE].

[63] CUORICINO collaboration, C. Arnaboldi et al., Results from a search for the $0 \nu \beta \beta$-decay of Te-130, Phys. Rev. C 78 (2008) 035502 [arXiv:0802.3439] [INSPIRE].

[64] NEMO collaboration, R. Arnold et al., First results of the search of neutrinoless double beta decay with the NEMO 3 detector, Phys. Rev. Lett. 95 (2005) 182302 [hep-ex/0507083] [INSPIRE].

[65] NEMO collaboration, A.S. Barabash and V.B. Brudanin, Investigation of double beta decay with the NEMO-3 detector, Phys. Atom. Nucl. 74 (2011) 312 [arXiv:1002.2862] [INSPIRE].

[66] F. del Aguila, J. de Blas and M. Pérez-Victoria, Effects of new leptons in electroweak precision data, Phys. Rev. D 78 (2008) 013010 [arXiv:0803.4008] [INSPIRE].

[67] A. Pierce and J. Thaler, Natural dark matter from an unnatural Higgs boson and new colored particles at the TeV scale, JHEP 08 (2007) 026 [hep-ph/0703056] [INSPIRE]. 
[68] R. Barbieri, L.J. Hall and V.S. Rychkov, Improved naturalness with a heavy Higgs: an alternative road to LHC physics, Phys. Rev. D 74 (2006) 015007 [hep-ph/0603188] [INSPIRE].

[69] L. Lopez Honorez and C.E. Yaguna, The inert doublet model of dark matter revisited, JHEP 09 (2010) 046 [arXiv: 1003.3125] [INSPIRE].

[70] A. Arhrib, Y.-L.S. Tsai, Q. Yuan and T.-C. Yuan, An updated analysis of inert Higgs doublet model in light of the recent results from LUX, PLANCK, AMS-02 and LHC, JCAP 06 (2014) 030 [arXiv: 1310.0358] [INSPIRE].

[71] G. Belanger, F. Boudjema and A. Pukhov, micrOMEGAs : a code for the calculation of Dark Matter properties in generic models of particle interaction, arXiv:1402.0787.

[72] ATLAS collaboration, Observation of a new particle in the search for the standard model Higgs boson with the ATLAS detector at the LHC, Phys. Lett. B 716 (2012) 1 [arXiv: 1207.7214] [INSPIRE].

[73] CMS collaboration, Observation of a new boson at a mass of $125 \mathrm{GeV}$ with the CMS experiment at the LHC, Phys. Lett. B $\mathbf{7 1 6}$ (2012) 30 [arXiv:1207.7235] [INSPIRE].

[74] LUX collaboration, D.S. Akerib et al., First results from the LUX dark matter experiment at the Sanford Underground Research Facility, Phys. Rev. Lett. 112 (2014) 091303 [arXiv: 1310.8214] [INSPIRE].

[75] MEG collaboration, J. Adam et al., New constraint on the existence of the $\mu^{+} \rightarrow e^{+} \gamma$ decay, Phys. Rev. Lett. 110 (2013) 201801 [arXiv: 1303.0754] [INSPIRE].

[76] BABAR collaboration, B. Aubert et al., Searches for lepton flavor violation in the decays $\tau^{ \pm} \rightarrow e^{ \pm} \gamma$ and $\tau^{ \pm} \rightarrow \mu^{ \pm} \gamma$, Phys. Rev. Lett. 104 (2010) 021802 [arXiv:0908.2381] [INSPIRE].

[77] SINDRUM II collaboration, W.H. Bertl et al., A search for muon to electron conversion in muonic gold, Eur. Phys. J. C 47 (2006) 337 [INSPIRE].

[78] A. Badertscher et al., New upper limits for muon - Electron conversion in sulfur, Lett. Nuovo Cim. 28 (1980) 401 [INSPIRE].

[79] SINDRUM II collaboration, C. Dohmen et al., Test of lepton flavor conservation in $\mu \rightarrow e$ conversion on titanium, Phys. Lett. B 317 (1993) 631 [INSPIRE].

[80] SINDRUM II collaboration, W. Honecker et al., Improved limit on the branching ratio of $\mu \rightarrow e$ conversion on lead, Phys. Rev. Lett. 76 (1996) 200 [INSPIRE].

[81] SINDRUM collaboration, U. Bellgardt et al., Search for the decay $\mu^{+} \rightarrow e^{+} e^{+} e^{-}$, Nucl. Phys. B 299 (1988) 1 [INSPIRE].

[82] K. Hayasaka et al., Search for lepton flavor violating $\tau$ decays into three leptons with 719 million produced $\tau^{+} \tau^{-}$pairs, Phys. Lett. B 687 (2010) 139 [arXiv:1001.3221] [INSPIRE]. 